\title{
A case of hepatoblastoma misdiagnosed as combined hepatocellular carcinoma and cholangiocarcinoma in an adult
}

Keun Woo Park', Chang Jin Seo', Dae Young Yun', Min Keun Kim, Byung Seok Kim', Young Seok Han ${ }^{2}$, Hoon Kyu Oh, and Chang Hyeong Lee ${ }^{1}$

Department of ${ }^{1}$ Internal Medicine, ${ }^{2}$ Surgery, and ${ }^{3}$ Pathology, Catholic University of Daegu School of Medicine, Daegu, Korea

Hepatoblastoma usually occurs in children under the age of 2 years, with very few cases reported in adults. We experienced a case of adult hepatoblastoma in a 36-year-old female with chronic hepatitis B . She had experienced sudden onset abdominal pain. Her serum alpha-fetoprotein level was markedly elevated, and abdominal CT showed a 9-cm mass with internal hemorrhage in the right hepatic lobe with hemoperitoneum, so an emergency hepatic central bisectionectomy was performed. The initial histologic examination revealed that the mass mimicked combined hepatocellular carcinoma and cholangiocarcinoma with spindle-cell metaplasia of the cholangiocarcinoma element. Follow-up abdominal CT performed 3 months later showed a $5.5-\mathrm{cm}$ metastatic mass in the left subphrenic area. Laparoscopic splenectomy with mass excision was performed, and hepatoblastoma was confirmed histologically. A histologic re-examination of previously obtained surgical specimens also confirmed the presence of hepatoblastoma. Metastatic hepatoblastoma was found at multiple sites of the abdomen during follow-up, and so chemotherapy with cisplatin, 5-fluorouracil (5-FU), and vincristine was applied, followed by carboplatin and doxorubicin . Despite surgery and postoperative chemotherapy, she died 12 months after symptom onset. (Clin Mol Hepatol 2015;21:300-308)

Keywords: Hepatoblastoma; Surgery; Chemotherapy

\section{INTRODUCTION}

Most cases of hepatoblastoma are found under age two, and occurrence in adults is extremely rare. ${ }^{1,2}$ There are only 45 reported cases of adult hepatoblastoma globally, ${ }^{3-5}$ and only 3 cases in Korea. ${ }^{2,6,7}$ Most of the cases are asymptomatic and might be diagnosed at late stages of the disease. ${ }^{2,8}$ Complete surgical resection is essential in the treatment of hepatoblastoma and standardized chemotherapy which reduces tumor size increases resectabilities, ${ }^{9}$ but not so many experiences in adult hepatoblastoma makes it difficult to occur complete remission. Survival time varies from 2 week to 38 months, with a median of 6 months and is shorter in adults than childhood. ${ }^{10}$ We report a case of adult hepatoblastoma with chronic hepatitis $B(C H B)$ which was misdiagnosed as a combined hepatocellular carcinoma and cholangiocarcinoma (cHCC-CC).

\section{Abbreviations:}

AFP, alpha-fetoprotein; CHB, chronic hepatitis B; CHCC-CC, combined hepatocellular carcinoma and cholangiocarcinoma; $\mathrm{HBV}$, hepatitis B virus; hCG, human chorionic gonadotropin; PET, positron emission tomography

\section{Corresponding author: Chang Hyeong Lee}

Department of Internal Medicine, Catholic University of Daegu School of Medicine, 17-33 Duryugongwon-ro, Namgu, Daegu 42472, Korea Tel: +82-53-650-4043, Fax: +82-53-623-7507

E-mail:chlee1@cu.ac.kr 


\section{CASE REPORT}

A 36-year-old female presented at the emergency department with aggravating right upper abdominal pain for 2 hours. The patient was diagnosed hepatitis B virus (HBV) carrier for several years and non-alcoholics. No other specific personal and familial medical history was noted. Initial blood pressure was 100/60 $\mathrm{mmHg}$, pulse rate $70 / \mathrm{min}$, respiration rate $20 / \mathrm{min}$, body temperature $37.5^{\circ} \mathrm{C}$. The laboratory findings were white blood cell 12,000/ $\mathrm{mm}^{3}$ (poly: $70 \%$ ), hemoglobin $12.8 \mathrm{~g} / \mathrm{dL}$, platelet $198,000 / \mathrm{mm}^{3}$, prothrombin time 14.3 seconds, international normalized ratio 1.11, aspartate aminotransferase $22 \mathrm{IU} / \mathrm{L}$, alanine aminotransferase $12 \mathrm{IU} / \mathrm{L}$, total bilirubin $0.5 \mathrm{mg} / \mathrm{dL}$, alkaline phosphatase 134
IU/L, lactate dehydrogenase $295 \mathrm{IU} / \mathrm{L}$, gamma-glutamyl transpeptidase $26 \mathrm{IU} / \mathrm{L}$, protein/albumin 6.4/4.0 g/dL, uric acid $5.0 \mathrm{mg} / \mathrm{dL}$, blood urea nitrogen/creatinine 16.6/0.7 mg/dL. And serum viral markers were $\mathrm{HBsAg}(+)$, anti-HBs $(-)$, anti-HBC (+), HBeAg (-), anti-HBe (+), HBV-DNA <20 IU/mL, anti-HCV (-) and anti-HIV (-). The patient's serum $\alpha$-fetoprotein (AFP) was $676.5 \mathrm{ng} / \mathrm{mL}$ and carbohydrate antigen 19-9 (CA19-9) <0.6 U/mL. She took an abdominal CT scan, which showed a $9 \mathrm{~cm}$ sized necrotic mass with internal hemorrhage at the right hepatic lobe and ruptured to peritoneum (Fig. 1A), so an emergency hepatic central bisectionectomy was done (Fig. 2A). The initial histological diagnosis was cHCC-CC with spindle cell metaplasia of cholangiocarcinoma element (Fig. 2B). The serum AFP was decreased to $7.67 \mathrm{ng} / \mathrm{mL}$ at
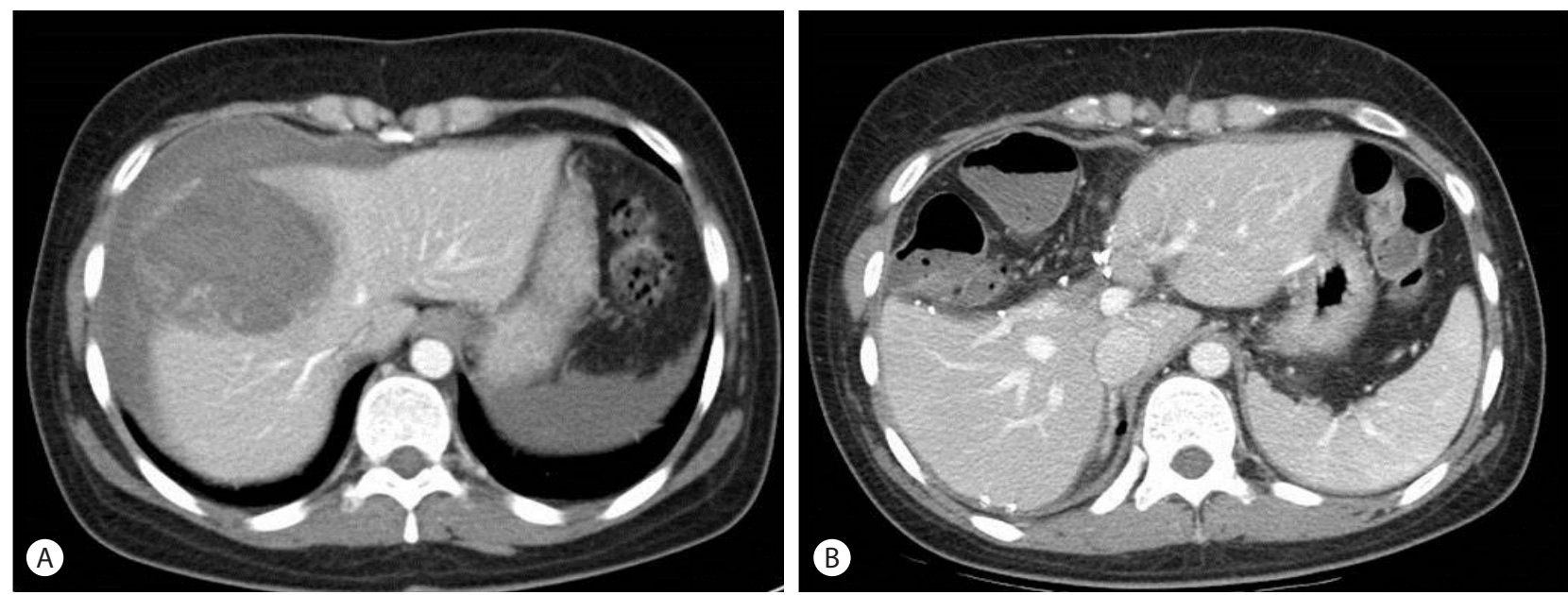

Figure 1. Abdominal CT scan. (A) A 9-cm necrotic mass with internal hemorrhage was evident in the right hepatic lobe that had ruptured into the peritoneum. (B) At 1 month after hepatic central bisectionectomy, no remnant lesion was found.
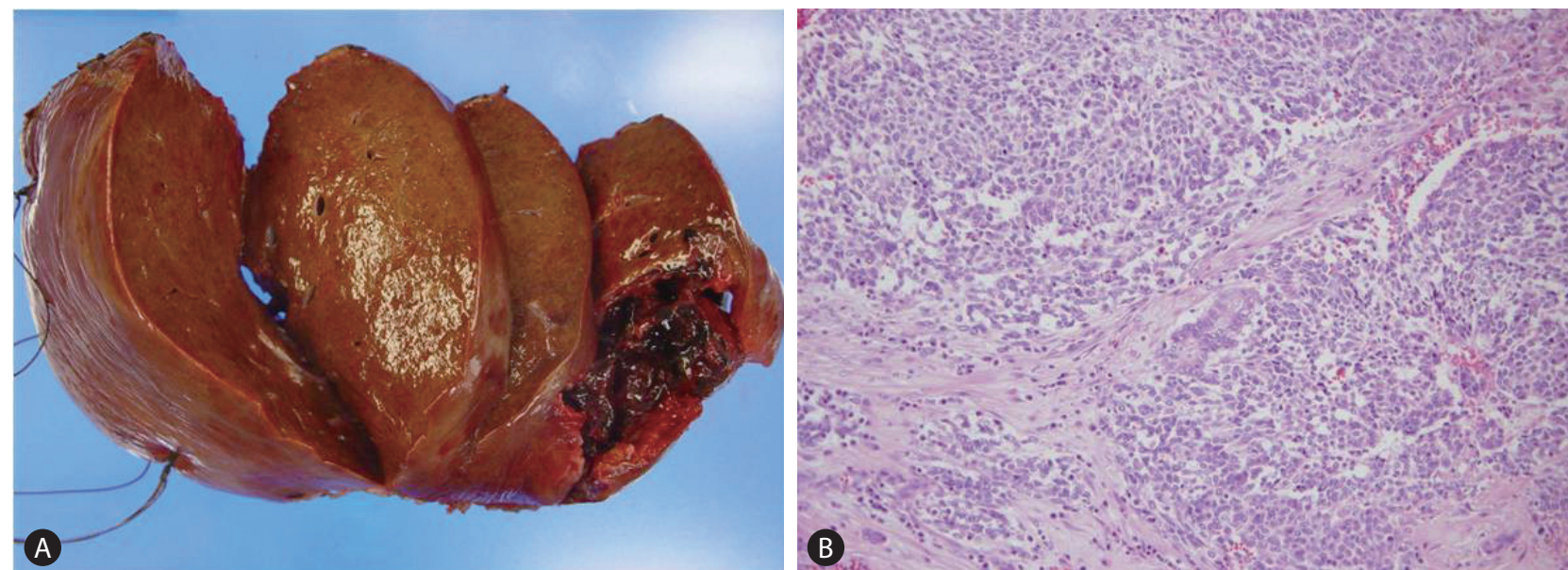

Figure 2. Pathologic features of the liver mass. (A) The resected liver shows a well-circumscribed hemorrhagic and necrotic mass in the right lobe in a background of micronodular cirrhosis. (B) Microscopy shows diffuse primitive cells with a trabecular growth pattern and gland formations (H\&E stain, $\times 100)$. 

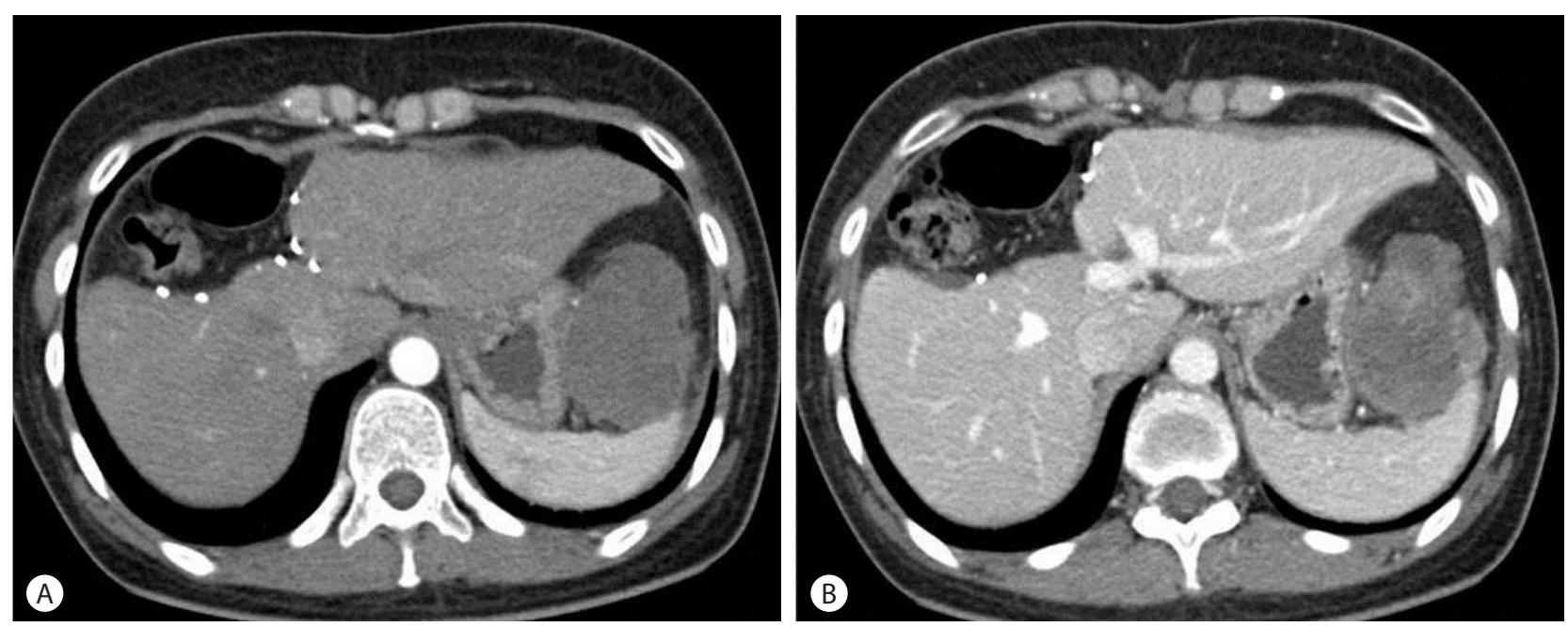

Figure 3. Follow-up abdominal CT scan obtained 3 months after resection. (A) A newly developed 5.5-cm left subphrenic mass was present (arterial phase). (B) The mass appeared with mild enhancement in the delayed image (portal phase).

time of discharge. Regarding as CHCC-CC, postoperative adjuvant chemotherapy with tegafur/uracil (UFT) was administrated for 3 months. 3 months later, follow-up abdominal CT scanning showed previously unseen a $5.5 \mathrm{~cm}$ sized left subphrenic mass with mild enhancement in delayed image (Fig. 3A, B) and AFP was increased to $312.06 \mathrm{ng} / \mathrm{mL}$. She underwent laparoscopic splenectomy with mass excision. On histologic examination, mesenchymal elements consisted of a proliferation of primitive-appearing mesenchymal spindle-shaped cells, intimately admixed with the epithelial elements in a highly cellular pattern. Cytoplasm was more abundant than that of mature fibroblasts, and the nucleus was elongated and plump. These cells blended progressively with areas of less intense cellular mesenchymal proliferation, and with relatively acellular, fibrous septa. Osteoid was present either within the primitive mesenchyme, near the fibrous septa or pseudocapsule, or admixed within the epithelial elements. Osteoid foci contained cells morphologically identical to osteoblasts (Fig. 4A). The immunohistochemistry stains showed expression of hepatocyte, B-HCG, AFP, vimentin, CK7, CK19, CD56 and B-catenin and negativity for CEA. (Fig. 4B, C, D, E, F, G). Metastatic hepatoblastoma was confirmed by histologic examination with immunohistochemistry stains, so immunohistochemistrically re-examination of previous surgical specimens was also confirmed as hepatoblastoma. Follow-up abdominal CT scan performed 1 month after reoperation showed a newly onset $1.7 \mathrm{~cm}$ sized subtle enhancing soft tissue mass in splenic bed, and positron emission tomography (PET) showed multiple FDG uptake(max SUV > 3.80) in left upper and lower quadrant area of abdomen, paralumbar area including right subphrenic area (Fig. 5A, B, C). Follow-up AFP was $162.69 \mathrm{ng} / \mathrm{mL}$.
Systemic chemotherapy started with cisplatin $\left(60 \mathrm{mg} / \mathrm{m}^{2}\right), 5$-fluorourasil $(5-\mathrm{FU})\left(600 \mathrm{mg} / \mathrm{m}^{2}\right)$, vincristine $\left(1.5 \mathrm{mg} / \mathrm{m}^{2}\right)$ and total 3 cycles of chemotherapy were done every 4 week. Entecavir 0.5 $\mathrm{mg}$ was also started for chemoprophylaxis of CHB. After the 3rd chemotherapy cycle, follow-up abdominal CT and PET scan showed progression of multiple intraperitoneal metastasis with large amount of intraperitoneal fluid (Fig. 6A, B) and follow-up AFP was further increased to $254 \mathrm{ng} / \mathrm{mL}$. So chemotherapy regimen was changed to carboplatin $\left(350 \mathrm{mg} / \mathrm{m}^{2}\right)$ with doxorubicin $\left(30 \mathrm{mg} / \mathrm{m}^{2}\right)$ every 3 weeks. The patient experienced neutropenia after the new regimen, but recovered shortly after treatment with granulocyte colony-stimulating factor (G-CSF). After second carboplatin with doxorubicin chemotherapy, follow-up AFP was increased $1510.19 \mathrm{ng} / \mathrm{mL}$, but abdominal CT scan showed that amount of ascites was decreased (Fig. 7). Because of radiologic improvements, third and fourth chemotherapy were performed. After 4th chemotherapy, follow-up abdominal CT revealed progression of multiple intraperitoneal metastatic masses, newly developed hepatic metastasis and large amount of intraperitoneal fluid (Fig. 8). A diagnostic paracentesis was performed and showed white blood cell $310 / \mathrm{mm}^{3}$ (poly $20 \%$, lymph $80 \%$ ), protein $3.6 \mathrm{~g} / \mathrm{dL}$ and albumin $2.3 \mathrm{~g} / \mathrm{dL}$ on ascitic fluid analysis. The serum albumin was $3.1 \mathrm{~g} / \mathrm{dL}$ and serum-ascites albumin gradient (SAAG) was $0.8 \mathrm{~g} /$ $\mathrm{dL}$. The AFB stain and culture were all negative in ascitic fluid. The AFP was $6881.93 \mathrm{ng} / \mathrm{mL}$ on serum. Regarded as peritoneal carcinomatosis, the patient underwent conservative treatments including the use of repeated therapeutic paracentesis for 1 month before death. 

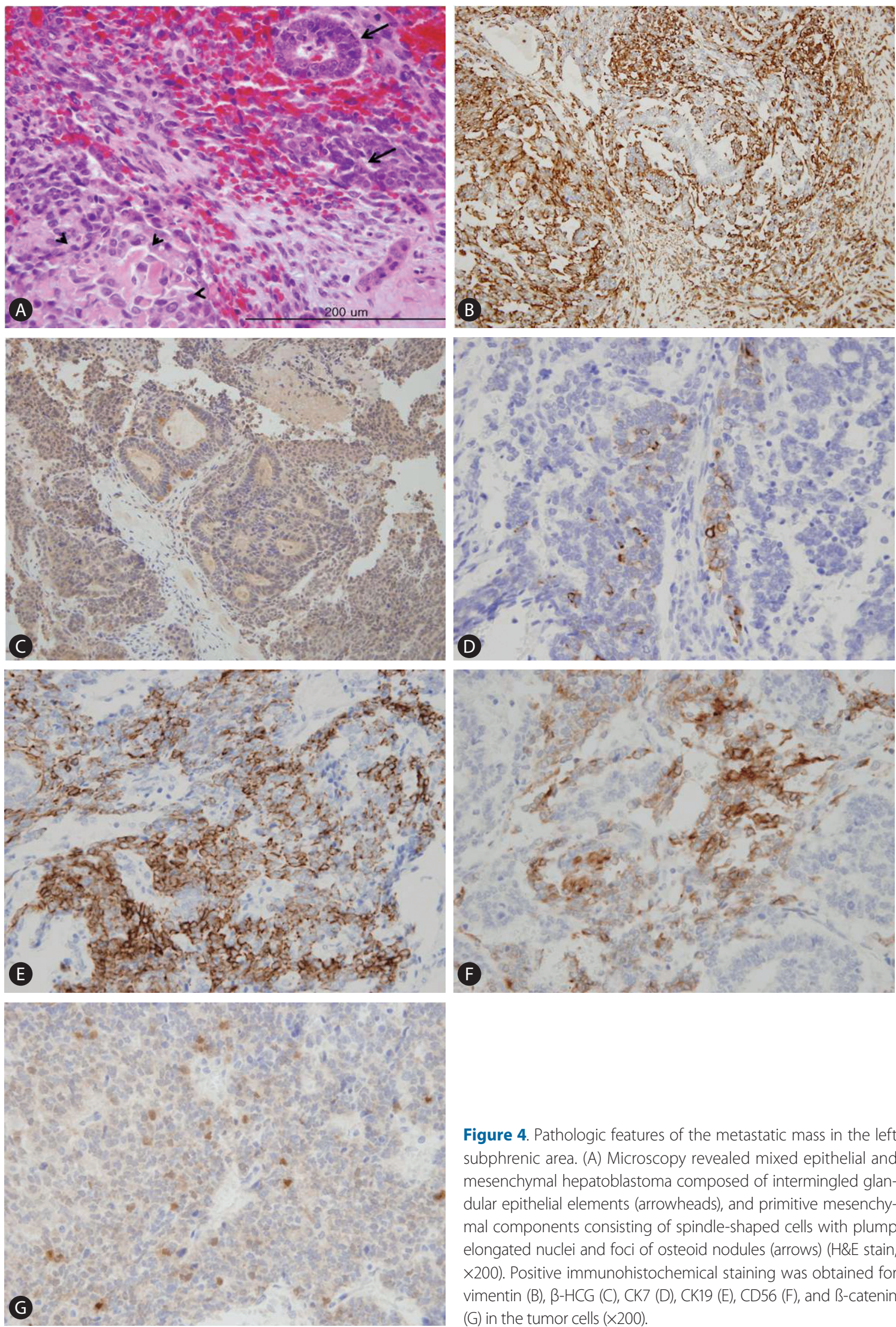

Figure 4. Pathologic features of the metastatic mass in the left subphrenic area. (A) Microscopy revealed mixed epithelial and mesenchymal hepatoblastoma composed of intermingled glandular epithelial elements (arrowheads), and primitive mesenchymal components consisting of spindle-shaped cells with plump elongated nuclei and foci of osteoid nodules (arrows) (H\&E stain, $\times 200$ ). Positive immunohistochemical staining was obtained for vimentin (B), $\beta$-HCG (C), CK7 (D), CK19 (E), CD56 (F), and ß-catenin $(G)$ in the tumor cells $(\times 200)$. 

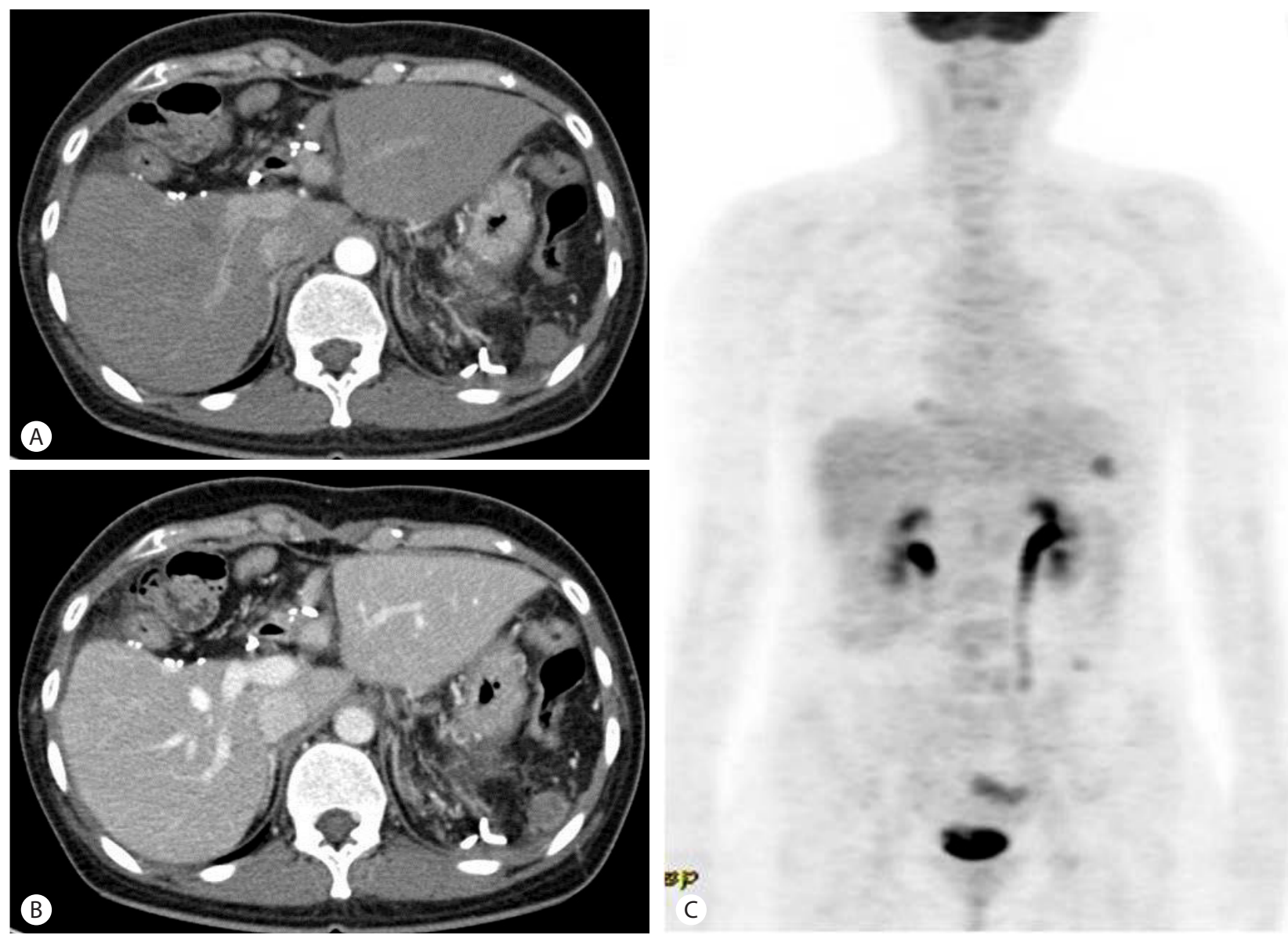

Figure 5. Follow-up CT scans in the arterial (A) and portal (B) phases and PET (C) scans obtained 1 month after the second resection. (A, B) A newly developed 1.7-cm subtle enhancing soft-tissue mass was evident in the splenic bed. (C) Multiple areas of FDG uptake (maximum SUV > 3.80) were present in the left upper and lower quadrants of the abdomen and in the paralumbar area including the right subphrenic area.

\section{DISCUSSION}

Hepatoblastoma is a highly malignant tumor occurring in infants, and reports of adult cases are extremely rare. The prognosis is poor because they are usually diagnosed in late stage. Initial symptoms are non-specific and the usual presentation is failure to thrive, loss of weight and a rapidly enlarging abdominal mass. It has been shown that chronic hepatitis or liver cirrhosis is often present in adult cases, whereas in children hepatoblastoma can occur in a normal liver. ${ }^{11}$ In our case, the patient was a HBV carrier. Because of the rare incidence of hepatoblastoma in adult, the initial consideration was a primary CHCC-CC rupture associated with CHB.

In Korea, 3 cases were already reported. Ahn et al., ${ }^{2}$ reported mixed hepatoblastoma in an adult. The patient's serum HBsAg was positive and AFP was $43,850 \mathrm{ng} / \mathrm{mL}$. Abdominal CT scan

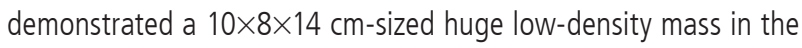

liver and selective celiac angiogram revealed faint tumor staining from hepatic artery and splenic artery. Based on the above findings, a clinical diagnosis of HCC was made. After surgery, this tumor was diagnosed as hepatoblastoma by H\&E stain. Microscopic examination revealed a tumor consisted of epithelial and mesenchymal elements. The mesenchymal cells were spindle in shape and proliferated over the whole tumor with focal osteosarcomatous differentiation. The epithelial components showed well-differentiated hepatocellular carcinoma-like areas, poorly differentiated acinar or tubular structures. The patient died about 2 months after operation. Shin et al., reported a case of hepatoblastoma in an adult. The patient's serum HBsAg was positive and AFP was 9,103 ng/mL. Abdominal CT scan demonstrated a $6 \times 7 \mathrm{~cm}$-sized huge low-density mass with central necrosis in liver. This mass was diagnosed as HCC on preoperative fine needle biopsy. After surgery, the mass was finally diagnosed as hepatoblastoma on H\&E stain. ${ }^{6}$ Lee et al., also reported a case of hepatoblastoma di- 

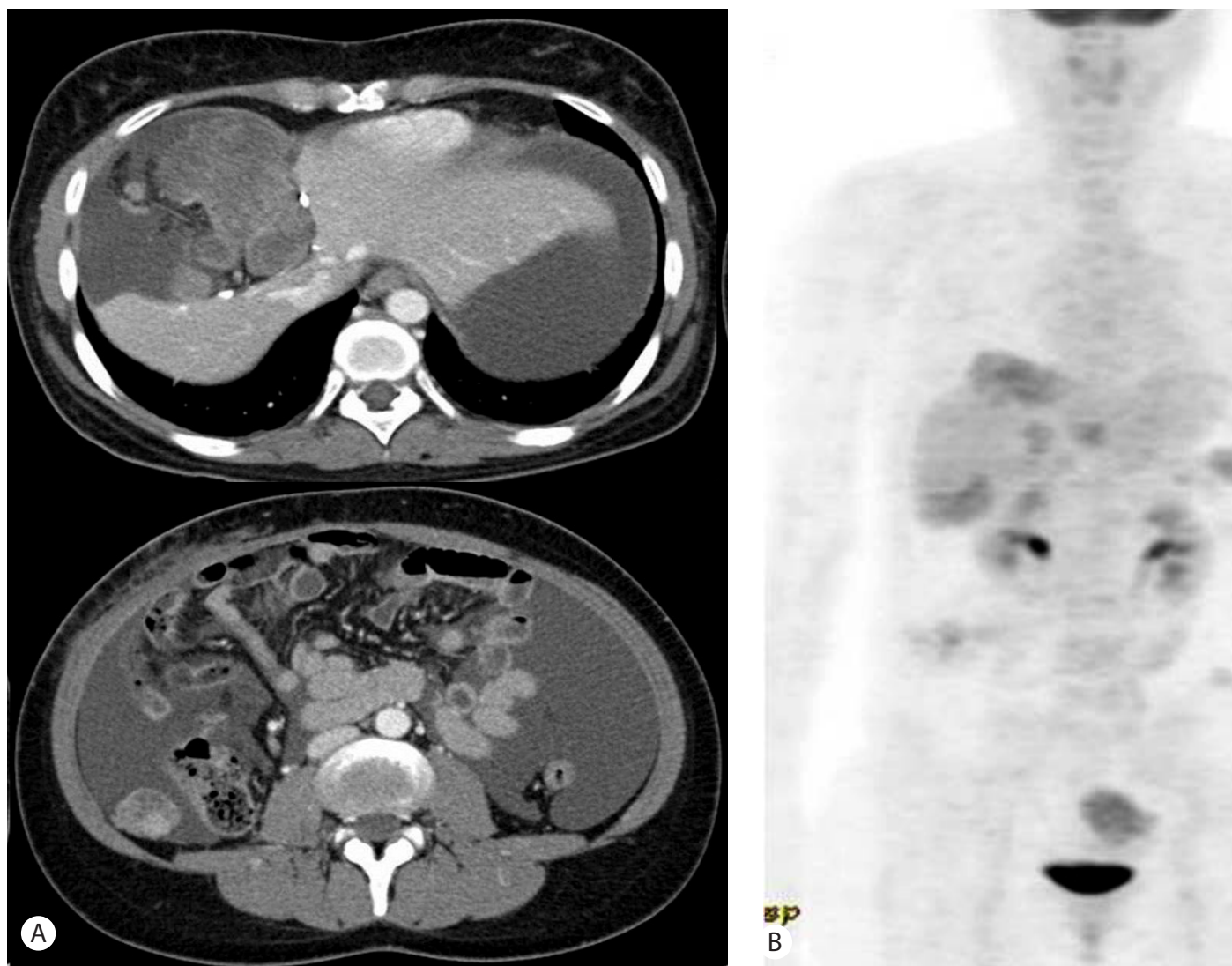

Figure 6. Follow-up CT (A) and PET (B) scans obtained after the third sessions of cisplatin, 5-FU, and vincristine chemotherapy. (A) Multiple intraperitoneal metastases with large amounts of intraperitoneal fluid were evident. (B) Multiple FDG uptake areas (maximum SUV > 4.46) were present in the subphrenic, subhepatic, and hepatorenal areas and in the left upper quadrant of the abdomen, both paracolic gutters, and the pelvic cavity.

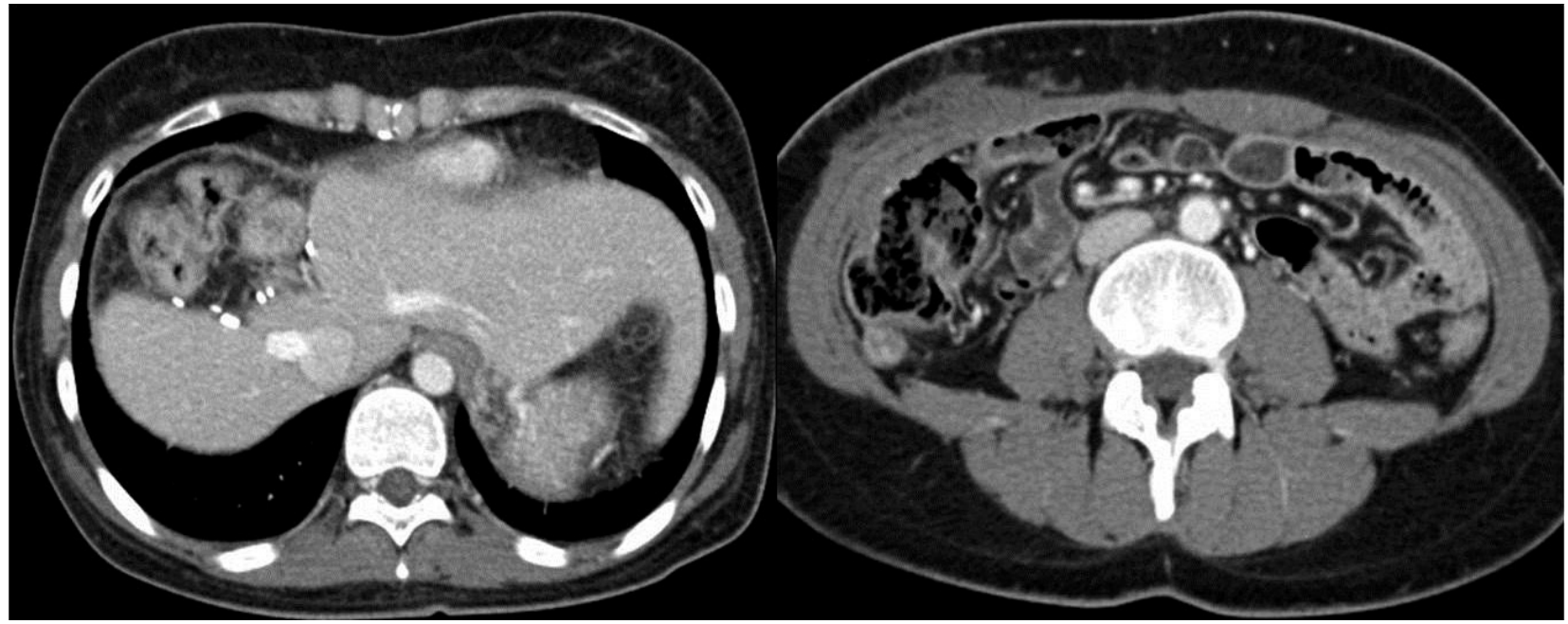

Figure 7. Abdominal CT scan obtained after the second carboplatin session with doxorubicin chemotherapy showing that the amount of ascites had decreased.

agnosed by H\&E stain in a pregnant woman. The patient's serum HBsAg was negative and AFP was $199 \mathrm{ng} / \mathrm{mL}$. Abdominal CT scan demonstrated a huge heterogeneous mass in the liver. After surgery, patient died in about 25 days. ${ }^{7}$ All of these cases were confirmed by H\&E stain. Our case was initially diagnosed primary CHCC-CC by H\&E stain. But, the mass was confirmed as a hepato- 


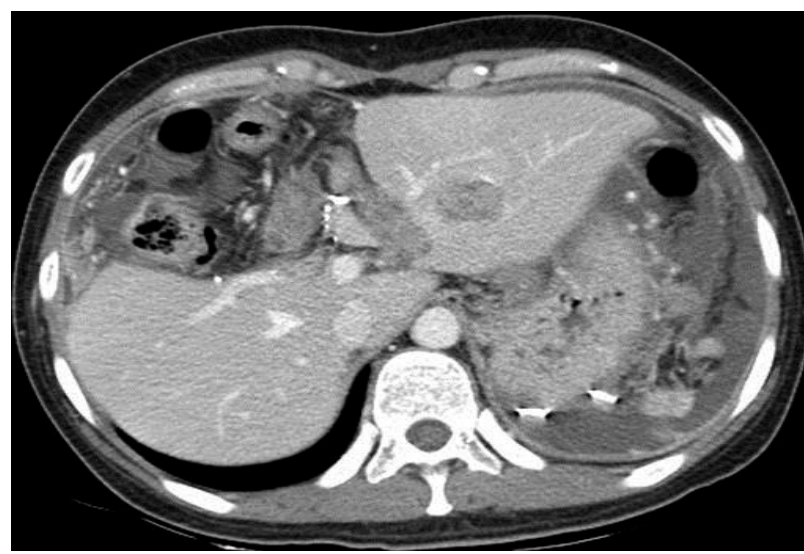

Figure 8. Abdominal CT scan obtained after the fourth carboplatin session with doxorubicin chemotherapy showing newly developed hepatic metastasis in the lateral segment, progression of multiple intraperitoneal metastatic masses, and an increased amount of ascites.

blastoma by histologic re-examination with immunohistochemistry stain.

According to the widely used classification of Ishak and Glunz, ${ }^{12}$ hepatoblastoma can be divided into mixed epithelial and mesenchymal type and epithelial type, and the latter can be divided into fetal type and embryonal type, ${ }^{13}$ but mixed type is more common. ${ }^{10}$ The pathogenesis of hepatoblastoma are not yet exactly discovered, but chromosome 1q, 2(or 2q), 4q, 8(or 8q) and 20 are known cytogenetic and molecular genetic alteration. ${ }^{14}$ Loss of heterozygosity imprinting at locus $11 p$ 15.5, nuclear p53 accumulation and p53 mutation are other suggested pathogenesis of the malignancy. ${ }^{15}$ High expression of polo-like kinase 1 may be related to poorer outcome, but need to get more research outcome. ${ }^{16} \mathrm{Ac}-$ cording to embryologic theory, hepatoblastoma is known to originate during intrauterine life, but this hypothesis seems difficult to apply in the adult. Because several cases of hepatoblastoma were found in more than 70 years of age ${ }^{3,11,17}$ and underlying liver cirrhosis was frequently found in adult, ${ }^{3,4}$ but not in children. So hepatoblastoma has a different pathogenic pathway in adult.

The mass size varies from $2.5 \mathrm{~cm}$ to $25 \mathrm{~cm}$ in diameter, more commonly presented as single mass in right lobe, so multifocal type was reported only 1 case..$^{10}$ In this case, presented with single mass in right lobe, AFP-producing mixed epithelial and mesenchymal type. Underlying fibrosis or cirrhosis was identified in 11 of 45 published hepatoblastoma adult cases. An additional 5 patients had a history of viral hepatitis (A, B or C), but no reported fibrosis.

General presenting symptoms are body weight loss, fever, abdominal pain, abdominal lymphadenopathy and masses, but these are non-specific, so found in a more advanced state. ${ }^{11}$

Imaging study plays an important role in the initial diagnosis. Ultrasonography can be used for initial evaluation, and CT, magnetic resonance imaging and laboratory examination like serum AFP, beta- human chorionic gonadotropin (hCG), serum platelet count also can be helpful. AFP is usually elevated, ${ }^{11}$ beta-hCG is rarely elevated but can be the cause of pediatric isosexual precocity. ${ }^{18}$ In the final diagnosis, histologic confirmation is essential to differentiate from hepatic sarcoma, carcinosarcoma, malignant mesenchymal tumor, HCC with sarcomatous change. ${ }^{11}$ Even if microscopic examination, differential diagnosis was difficult. 11,19,20 Especially, CHCC-CC which are intimately admixed HCC and CC was more difficult to distinguish from hepatoblastoma by histologic morphology. ${ }^{12}$ Actually, Gonzalez-Crussi et al., reported that pathologists_were unfamiliar with various epithelial hepatoblastoma, so they mistook hepatoblastoma for hepatocellular carcinoma in adult. ${ }^{21}$ Because the lack of experience of hepatoblastomas in adult patients with $\mathrm{CHB}$, we mistook it for CHCC-CC initially. When the diagnosis of hepatoblastoma was difficult, immunohistochemical stain has been used. The reactivity has been found for keratin, EMA, vimentin, polyclonal CEA, HepPar-1, AFP, $\alpha_{1}{ }^{-}$ antitrypsin, CD99, CD56(N-CAM), human chorionic gonadotropin, transferrin receptor, and delta-like protein. ${ }^{22,23}$ There was also showed that aberrant nuclear immunostaining for $\beta$-catenin is a highly characteristic feature. ${ }^{24}$ Our case showed that positive immunohistochemical stains of vimentin, $\beta$-HCG, AFP, CK7, CK19, CD56 and B-catenin.

Complete surgical resection is essential in the treatment of hepatoblastoma. But survival improvement in last 30 years has been due to not only the surgery itself, but standardized chemotherapy which reduces tumor size and increases resectabilities. ${ }^{9}$ In 1990 1994, SIOPEL-1 (Society of Pediatric Oncology Liver Tumor Study Group launched its first prospective trial) revealed preoperative chemotherapy and delayed surgical resection increases tumor resectability. ${ }^{25}$ Doxorubicin, cisplatin, vincristine, 5-FU, cyclophosphamide are known relatively well responding chemotherapeutic agents. ${ }^{26}$ Radical resection and chemotherapy are known most appropriate approach for hepatoblastoma, but not so many experiences in adult hepatoblastoma makes it more urgent to establish determinant of prognosis in hepatoblastoma. ${ }^{27}$ Suggested poor prognostic factors were multiple lobes involvement, embryonic type, decreased P27 gene expression, multifocal dissemination, AFP level lower than 100 or higher than $100,000 \mathrm{ng} / \mathrm{mL}^{28,29}$ Survival time varies from 2 weeks to 38 months, 6 months in median, shorter in adult than childhood. ${ }^{10}$ The 3 reported cases in Korea, 
survival time of 2 cases were about 25 days and 2 months after surgery. ${ }^{2,7}$ The one case could not be identified. ${ }^{6}$ But, our case showed that survival time was extended 12 months after the cisplatin, 5-FU, vincristine of 3 cycles chemotherapy and carboplatin, doxofubicin of 4 cycles chemotherapy. In addition, our case was quite severe that the size of tumor was $9 \mathrm{~cm}$ and intraperitoneal metastasis from a ruptured hepatoblastoma compared to the previous three cases, however, aggressive chemotherapy improved survival time.

In conclusion, hepatoblastoma in adults is rare, but must be a consideration. It shows no distinct histological findings, but the diagnosis requires a pathologic examination including immunohistochemical stain. Radical resection and active chemotherapy may improve survival from adult hepatoblastoma.

\section{Conflicts of Interest}

The authors have no conflicts to disclose.

\section{REFERENCES}

1. Lack EE, Neave C, Vawter GF. Hepatoblastoma. A clinical and pathologic study of 54 cases. Am J Surg Pathol 1982;6:693-705.

2. Ahn HJ, Kwon KW, Choi YJ, Kim HJ, Hong SP, Oh D, et al. Mixed hepatoblastoma in an adult--a case report and literature review. J Korean Med Sci 1997;12:369-373.

3. Kasper HU, Longerich T, Stippel DL, Kern MA, Drebber U, Schirmacher P. Mixed hepatoblastoma in an adult. Arch Pathol Lab Med 2005;129:234-237.

4. Yamazaki M, Ryu M, Okazumi S, Kondo F, Cho A, Okada T, et al. Hepatoblastoma in an adult:A case report and clinical review of literatures. Hepatol Res 2004;30:182-188.

5. Parada LA, Bardi G, Hallén M, Hägerstrand I, Tranberg KG, Mitelman $F$, et al. Cytogenetic abnormalities and clonal evolution in an adult hepatoblastoma. Am J Surg Pathol 1997;21:1381-1386.

6. Shin DH, Kim YS, Yoon MH, chun BK, Kim YO, Hur B, et al. A case of hepatoblastoma in adult. Korean $\mathrm{J}$ Hepatobiliary Pancreat Surg 1999:3:169-173.

7. Lee CH, Kim CS, Ju KJ, Park SM, Shim YW, Song KY, et al. A Case of Hepatoblastoma in A Pregnant Woman. Korean J Gastroenterol 1993:25:1366-1369.

8. Remes-Troche JM, Montaño-Loza A, Meza-Junco J, García-Leiva J, Torre-Delgadillo A. Hepatoblastoma in adult age. A case report and literature review. Ann Hepatol 2006:4:179-181.

9. Pimpalwar AP, Sharif K, Ramani P, Stevens M, Grundy R, Morland B, et al. Strategy for hepatoblastoma management: Transplant versus nontransplant surgery. J Pediatr Surg 2002;37:240-245.
10. Zheng MH, Zhang L, Gu DN, Shi HQ, Zeng QQ, Chen YP. Hepatoblastoma in adult: review of the literature. J Clin Med Res 2009;1:1316.

11. Oda H, Honda K, Hara M, Arase Y, Ikeda K, Kumada H. Hepatoblastoma in an 82-year-old man: an autopsy case report. Acta Pathol Jpn 1990;40:212-218.

12. Ishak KG, Glunz PR. Hepatoblastoma and hepatocarcinoma in infancy and childhood. Report of 47 cases.Cancer 1967;20:396-422.

13. Sugino K, Dohi K, Matsuyama T, Asahara T, Yamamoto M. A case of hepatoblastoma occurring in an adult. Jpn J Surg 1989;19:489-493.

14. Nagata $T$, Nakamura $M$, Shichino $H$, Chin M, Sugito $K$, Ikeda $T$, et al. Cytogenetic abnormalities in hepatoblastoma: report of two new cases and review of the literature suggesting imbalance of chromosomal regions on chromosomes 1, 4, and 12. Cancer Genet Cytogenet 2005;156:8-13.

15. Prange W, Breuhahn K, Fischer F, Zilkens C, Pietsch T, Petmecky K, et al. Beta-catenin accumulation in the progression of human hepatocarcinogenesis correlates with loss of E-cadherin and accumulation of p53, but not with expression of conventional WNT-1 target genes. J Pathol 2003;201:250-259

16. Yamada S, Ohira M, Horie $H$, Ando $K$, Takayasu $H$, Suzuki $Y$, et al. Expression profiling and differential screening between hepatoblastomas and the corresponding normal livers: identification of high expressionof the PLK1 oncogene as a poor-prognostic indicator of hepatoblastomas. Oncogene 2004;5:5901-5911.

17. Carter R. Hepatoblastoma in the adult. Cancer 1969;23:191-197.

18. Sotelo-Avila C, Gonzalez-Crussi F, Fowler JW. Complete and incomplete forms of Beckwith-Wiedemann syndrome: their oncogenic potential. J Pediatr 1980;96:47-50.

19. Blanding JD. Mixed malignant tumor of the liver in an adult. Case report and review of some features. Arch Pthol 1968;86:108-110

20. Ludwig J, Grier MW, Hoffman HN Jr, McGill DB. Calcified mixed malignant tumor of the liver. Arch Pathol 1975;99:162-166.

21. González-Crussi F, Upton MP, Maurer HS. Attempt at characterization of histologic subtypes. Am J Surg Pathol 1982;6:599-612.

22. Abenoza P, Manivel JC, Wick MR, Hagen K, Dehner LP. Hepatoblastoma: An immunohistochemical and ultrastructural study. Hum Pathol 1987;18:1025-1035

23. Ramsay $A D$, Bates $A W$, Williams $S$, Sebire NJ. Variable antigen expression in hepatoblastomas. Appl Immunohistochem Mol Morphol 2008;16:140-147.

24. Blaker H, Hofmann WJ, Rieker RJ, Penzel R, Graf M, Otto HF. Betacatenin accumulation and mutation of the CTNNB1 gene in hepatoblastoma. Genes Chromosomes Cancer 1999;25:399-402.

25. Zsíros J, Brugières L, Brock P, Roebuck D, Maibach R, Child M, et al. Efficacy of irinotecan single drug treatment in children with refractory or recurrent hepatoblastoma - A phase II trial of the childhood liver tumour strategy group (SIOPEL). Eur J Cancer 2012;48:3456- 
3464.

26. Reynolds M. Pediatric liver tumors. Semin Surg Oncol 1999;16:159172.

27. Sugawara W, Haruta M, Sasaki F, Watanabe N, Tsunematsu Y, Kikuta $A$, et al. Promoter hypermethylation of the RASSF1A gene predicts the poor outcome of patients with hepatoblastoma. Pediatr Blood Cancer 2007;49:240-249.
28. Mukhopadhyay P, Kundu SS, Banerjee A, Mukherjee A. Adult hepatoblastoma in a female Down's. J Assoc Physicians India 2007:55:242-243.

29. Brotto M, Finegold MJ. Distinct patterns of p27/KIP 1 gene expression in hepatoblastoma and prognostic implications with correlation before and after chemotherapy. Hum Pathol 2002;33:198-205. 